\title{
L'évolution du discours sur les ressources humaines en tourisme : entre théories et pratiques managériales
}

The evolution of the discourse on human resources in tourism: between managerial theories and practices

Boualem Kadri, Mohamed Reda Khomsi et Cyril Martin

\section{(2) OpenEdition}

\section{Journals}

Édition électronique

URL : http://journals.openedition.org/tourisme/2429

DOI : 10.4000/tourisme.2429

ISSN : 2492-7503

Éditeur

Éditions touristiques européennes

Référence électronique

Boualem Kadri, Mohamed Reda Khomsi et Cyril Martin, «L'évolution du discours sur les ressources humaines en tourisme : entre théories et pratiques managériales », Mondes du Tourisme [En ligne], 16 | 2019, mis en ligne le 01 décembre 2019, consulté le 09 avril 2020. URL : http://

journals.openedition.org/tourisme/2429 ; DOI : https://doi.org/10.4000/tourisme.2429

Ce document a été généré automatiquement le 9 avril 2020.

Mondes du tourisme est mis à disposition selon les termes de la licence Creative Commons Attribution - Pas d'Utilisation Commerciale - Pas de Modification 4.0 International. 


\title{
L'évolution du discours sur les
} ressources humaines en tourisme : entre théories et pratiques managériales

\author{
The evolution of the discourse on human resources in tourism: between \\ managerial theories and practices
}

Boualem Kadri, Mohamed Reda Khomsi et Cyril Martin

\section{Introduction}

1 L'entreprise, acteur majeur dans la société aujourd'hui, fait de plus en plus l'objet d'une attention particulière de la part des chercheurs et des acteurs politiques et sociaux : elle s'organise, s'adapte, apprend et marque son implication par une responsabilité sociale. Ce processus évolutif et adaptatif de l'organisation à la société montre un management postindustriel qui donne avantage non à la rationalité (technologie, organisation rationalisée) mais à la dimension de la ressource humaine de l'entreprise (Crozier, 1989). Peretti (2013, p. 6) résume ainsi l'évolution de cette dimension :

La fonction personnelle a émergé lentement dès la première moitié du $\mathrm{XX}^{\mathrm{e}}$, s'est professionnalisée dans la seconde moitié, est devenue fonction des ressources humaines et est reconnue comme une fonction stratégique aujourd'hui.

Depuis les années 2000, la pratique des ressources humaines devient "partenaire", s'adapte au contexte et aux défis (contingence de l'environnement) et s'ancre, pour l'horizon 2020, dans la société (responsabilité sociale, développement durable, humanitaire) (Peretti, 2013, p. 17).

S'agissant du tourisme, on note aussi que cette activité a évolué depuis les années 1970, d'une situation qualifiée d'éphémère (animations réalisées en fonction de la présence temporaire des touristes, pratiques économiques peu planifiées) à celle où l'activité 
devient une fonction stratégique, notamment pour l'économie urbaine et le développement territorial, ainsi qu'un "genre commun " pour la société (Lussault et Stock, 2007). Mais cette évolution est-elle aussi constatée en matière de gestion des ressources humaines de l'entreprise touristique?

L'irruption de nouvelles activités, dans l'économie traditionnelle des années 1970, tels les services et notamment ceux liés au tourisme (loisirs, divertissement, événements), résulte en une réalité économique et managériale confrontée à divers aspects: l'importance des relations humaines dans la confection du produit, la dimension d'intangibilité du produit, une gestion des ressources humaines différente, dans laquelle la prestation du personnel est une partie intégrante de la qualité du produit et de l'expérience humaine. Par ailleurs, une autre réalité vient marquer l'évolution du tourisme, notamment en ce qui concerne la construction de son identité scientifique, qui s'édifie peu à peu par la confrontation interdisciplinaire nécessaire à la compréhension de la complexité du phénomène touristique. Nous nous intéressons ici au discours en gestion des ressources humaines en tourisme, et plus particulièrement à son évolution dans le développement du tourisme et de l'entreprise. Si le discours scientifique sur cette complexité touristique se développe, en est-il de même du discours concernant la gestion des ressources humaines? Ce dernier évolue-t-il de la même manière que celui observé dans le management de l'organisation en général ? Comment se présente le discours en gestion des ressources humaines dans la recherche en tourisme, notamment dans les ouvrages et les revues? Pour répondre à ces interrogations, le présent article propose une analyse de type épistémologique du discours sur la gestion des ressources humaines en tourisme, constituée à partir d'articles issus de la revue Espaces tourisme \& loisirs. En sus d'être francophone, le choix de cette revue est motivé, d'une part, par le fait qu'elle est considérée par plusieurs acteurs du tourisme comme un espace privilégié pour exprimer leurs regards sur différents sujets qui touchent leur secteur et, d'autre part, par la possibilité offerte par la revue de mener une analyse diachronique sur les articles qui traitent de la gestion des ressources humaines en tourisme depuis les années 1990.

\section{Revue de littérature}

5 Pour saisir l'évolution du discours sur la gestion des ressources humaines en tourisme, nous avons lancé une recherche dans les principales bases de données scientifiques francophones (Cairn.info, OpenEdition, Persée, Tel [thèses en ligne] et Emerald Insight), indexées par le moteur de recherche Google Scholar, en utilisant des mots clés assez génériques comme: gestion des ressources humaines, GRH, tourisme, entreprise touristique. À ce titre, les résultats des différentes requêtes recensent un total de 3550 références publiées entre 2000 et 2019, réparties sur 99 pages. Au-delà de la dimension quantitative, deux constats importants peuvent être tirés des résultats de cette recherche :

- Même si notre objectif n'était pas de recenser des livres ou des manuels scolaires qui traitent de la gestion des ressources humaines en tourisme, il est intéressant de souligner qu'il n'existe aucun livre dédié exclusivement au sujet et référencé sur le moteur de recherche. Cette dimension se retrouve cependant dans plusieurs ouvrages qui traitent de la gestion des entreprises touristiques et elle occupe souvent un chapitre à part entière. Nonobstant cette existence, les problématiques abordées traitent dans la grande majorité de cas 
d'adaptation des techniques de gestion des ressources humaines au contexte touristique eu égard aux caractéristiques spécifiques de ce secteur.

- Pour ce qui est des articles scientifiques, sur les 3550 références répertoriées sur Google Scholar, uniquement trois articles abordent la question de l'évolution de la gestion de ressources humaines en tourisme. Pour les autres articles, la dimension touristique est soit absente, soit marginale, dans la mesure où le tourisme est cité uniquement pour illustrer des problèmes reliés souvent aux questions d'employabilité (recrutement, formation, adaptation culturelle, etc.).

6 Tenant compte de la faiblesse du corpus scientifique dédié à l'étude de l'évolution de la gestion des ressources humaines en tourisme, nous avons jugé important de retracer l'évolution de cette pensée GRH dans les manuels et les ouvrages généraux, dans la perspective de dégager une trame historique qui pourrait guider notre lecture des textes analysés dans la revue Espaces tourisme \& losirs.

\subsection{De la gestion de la force de travail à la gestion des talents}

7 L'observation de la littérature en management de l'organisation fait ressortir des efforts importants sur le plan scientifique : rapprochement entre sciences humaines de gestion, prise en compte des problématiques engendrées par les divers environnements, rupture avec la vision organiciste de l'entreprise et importance d'asseoir un discours managérial où la fonction stratégique des ressources humaines est présente. Dès 1987, Juan et Jacob observaient un effort d'intégration des sciences de la gestion et des sciences humaines dans les nouvelles conceptions managériales, soulignant par ailleurs que la transmission des connaissances portant sur la dimension humaine n'était pas unanimement reconnue, mais que la tentation de son contrôle et de son encadrement était toujours présente. Aujourd'hui, cette dimension occupe une place importante dans les théories du comportement organisationnel et constitue un pilier de la réflexion théorique dans le domaine du management. Ce postulat est aussi observable à la consultation de la table des matières d'ouvrages de management organisationnel où la question des ressources humaines est omniprésente. L'analyse des thématiques traitées sous ce volet au cours des quarante dernières années permet de conclure à un changement radical du paradigme qui soutient la pensée scientifique dans ce domaine de recherche. À titre d'illustration, déjà en 1994, Côté, Bélanger et Jacques constataient qu'en à peine quelques décennies, on est passé d'une vision «mercantiste », où l'individu est considéré comme une force de travail, à une vision bureaucratique, où le comportement des individus devra être uniformisé dans un souci de productivité, puis finalement à une vision organiciste où l'entreprise s'adapte aux besoins de l'individu.

Dans un contexte de mobilité internationale de la main-d'œuvre, de nouveaux défis apparaissent pour les entreprises, dont celles en tourisme et hôtellerie, nous faisant observer deux problématiques: d'une part, celle de l'adaptation de l'individu à l'organisation et au travail (culturelle) et, d'autre part, l'intégration des nouvelles technologies dans toutes les sphères de l'organisation. Selon Robbins et Judge (2011), dans un contexte où les entreprises opèrent sur plusieurs continents, les équipes de gestion sont appelées à collaborer sur certains dossiers de façon permanente, voire instantanée. Les nouvelles technologies permettent de dépasser les barrières spatiotemporelles en mettant en lien des équipes situées un peu partout à travers la 
planète, mais amènent en même temps de nouvelles problématiques en matière d'organisation du travail (travail en réseau, travail à distance, etc.).

La nécessité d'une adaptation aux contextes local et international conduit à l'émergence de nouveaux rôles et fonctions des ressources humaines dans l'entreprise, mis en évidence dans la littérature académique. Ainsi, selon Dupont (2013), la fonction qui doit être attribuée aux ressources humaines (RH) fait l'objet de nombreux discours quant au rôle qu'elles pourraient exercer au sein des entreprises et aux défis qu'elles devraient pouvoir relever. Dans les années 1990, des recherches effectuées en Europe montrent la valorisation de la fonction du DRH (développement organisationnel) et l'importance accordée à la fonction de DRH (position stratégique) au sein de l'entreprise (Bournois, Rojot et Scaringella, 2003).

En embrassant ce développement stratégique, un DRH doit faire preuve d'une grande polyvalence s'agissant du fonctionnement de l'entreprise, de façon à se positionner en tant que réel acteur stratégique de son organisation. Ses fonctions pourraient être «hyper-partagées» (Guérin et Pigeyre, 2007) par le transfert de certaines activités aussi bien vers l'extérieur que vers l'intérieur de l'entreprise.

\subsection{En tourisme, la GRH est en mode d'adaptation continue}

11 Le tourisme est présenté par les organismes internationaux comme une des activités les plus performantes dans le monde (plus de $9 \%$ du PIB mondial), qui comporte aussi divers aspects la distinguant des autres activités économiques, ce que font aussi les ouvrages en tourisme (généraux et spécialisés). Dans les ouvrages généraux, on remarque que l'entreprise et la gestion des ressources humaines sont présentées à travers notamment les problèmes rencontrés par l'activité et les pratiques développées. Mesplier et Bloc-Duraffour (2014) donnent quelques informations sur le lien entre tourisme et emploi, en montrant son importance économique tout en précisant la difficulté à l'évaluer (emplois directs-indirects, consommation trans-sectorielle, etc.). Stock et ses collègues (2003), abordent aussi le tourisme et ses retombées économiques pour la société (revenus, investissements) et les marchés (alliances, types d'emplois); ils montrent la complexité et la diversité de l'activité seulement au niveau de la société, sans évoquer l'entreprise. Copper et Hall, dont le livre sur le tourisme international (Contemporary Tourism : an International Approach) a été traduit en français, présentent cette activité suivant une approche davantage managériale, tout en apportant une critique du discours scientifique en tourisme (problème de définition des notions). Ces auteurs font en effet observer la difficulté de mesure dans l'industrie touristique, qui serait due à la méconnaissance de l'offre et donc à la complexité de l'industrie : taille diverse des entreprises, secteurs disparates, cultures en compétition (public, privé) pas de produit unique mais des biens et services aux aspects tangibles et intangibles (Copper et Hall, 2008). Ils écrivent que la ressource humaine est importante pour la qualité du produit, mais recèle un problème : «le tourisme de ce début de $\mathrm{XXI}^{\mathrm{e}}$ est marqué par une crise des ressources humaines", caractérisée par la mobilité de la main-d'œuvre, la nécessité de pratiques managériales interculturelles, la démographie en baisse dans les pays développés, une fonction des ressources humaines faible dans les petites entreprises, etc. (ibid. : 177).

Dans l'objectif de retracer l'évolution du discours sur la gestion stratégique des ressources humaines en tourisme, restauration et hôtellerie dans la littérature, Madera 
et ses collègues (2017) ont analysé 300 articles publiés au cours des vingt dernières années dans neuf revues scientifiques classées dans le premier tiers des revues en tourisme et hôtellerie. Leurs analyses, du contenu et des thématiques, les amènent à conclure, entre autres, qu'il existe une corrélation significative entre la performance des ressources humaines et celle des organisations. En d'autres termes, ces auteurs constatent que dans la grande majorité des articles analysés, les chercheurs ont tendance à établir un lien entre l'amélioration des conditions de travail des employés et les gains de performance organisationnelle. En effet, les caractéristiques spécifiques du secteur touristique (pénurie de main-d'œuvre, saisonnalité, mobilité internationale et servuction) conduisent les chercheurs et les professionnels à s'intéresser davantage à ce type de problématique afin de proposer des pratiques bénéfiques pour l'ensemble des acteurs du secteur.

Le constat de Madera et ses collègues (2017) est confirmé aussi par d'autres auteurs qui se sont intéressés aux problématiques de la gestion des ressources humaines en tourisme. C'est le cas notamment de Monereau (2008), Lozato-Giotart, Leroux et Balfet (2012), Leroux et Pupion (2014) et Clergeau et al. (2014). Ces auteurs francophones, qui ont consacré des livres ou des chapitres de livre à la question de la GRH en tourisme, retracent l'évolution de la culture d'entreprise vis-à-vis des ressources humaines au cours du XXe siècle. À ce titre, Monereau (2008) présente ces dernières comme un gisement de compétences qu'il faudra bien utiliser pour améliorer la performance de l'entreprise, alors que Leroux et Pupion (2014) expliquent que la modification de l'environnement externe et interne des entreprises touristiques les a forcées à s'adapter en matière de gestion de ressources humaines afin de rester compétitives. Nous retrouvons le souci d'adaptation à un environnement en perpétuelle mutation chez des auteurs anglo-saxons comme Goeldner et Ritchie (2009), Lee-Ross et Pryce (2010) et Cook, Hsu et Marqua (2014). De façon presque unanime, ces auteurs soutiennent que l'accessibilité grandissante des destinations et des attractions touristiques a poussé les entreprises qui œuvrent dans ce domaine à revoir leurs façons de faire, plus particulièrement au niveau de la gestion des ressources humaines. À titre d'illustration, Lee-Ross et Pryce (2010) identifient le management interculturel comme un enjeu important du XXI siècle. Pour eux, la diversité des employés et des touristes exige une prise en considération de la dimension culturelle pour que les deux parties soient satisfaites de la prestation de service.

14 Finalement, comme on peut le constater dans la figure ci-dessous, l'évolution du discours en gestion des ressources humaines, que ce soit en tourisme ou de façon générale, a suivi pratiquement le même cheminement. Autrement dit, la perspective pratique et professionnelle a dominé l'appréhension scientifique du discours et cela s'explique par divers facteurs: le développement récent du tourisme et de son industrie, une activité dépeinte comme complexe et difficile à appréhender, un tourisme perçu à travers des retombées économiques rapides en termes de revenus nécessitant le recours à des pratiques professionnelles. L'importance accordée à la gestion des ressources humaines est donc tardive ; par exemple, on remarque dans un ouvrage des années 1990, Économie et management (Tinard, 1992), que l'auteur met l'accent sur les métiers du tourisme et leurs problèmes, ainsi que sur les aspects économiques (offre, demande, produit); l'hôtel y est ainsi perçu à partir des perceptions des touristes et des marchés, non à partir de la gestion de la ressource humaine. 
Figure 1. Évolution de la fonction RH (1960-2010) dans le discours scientifique et son déploiement dans l'entreprise touristique

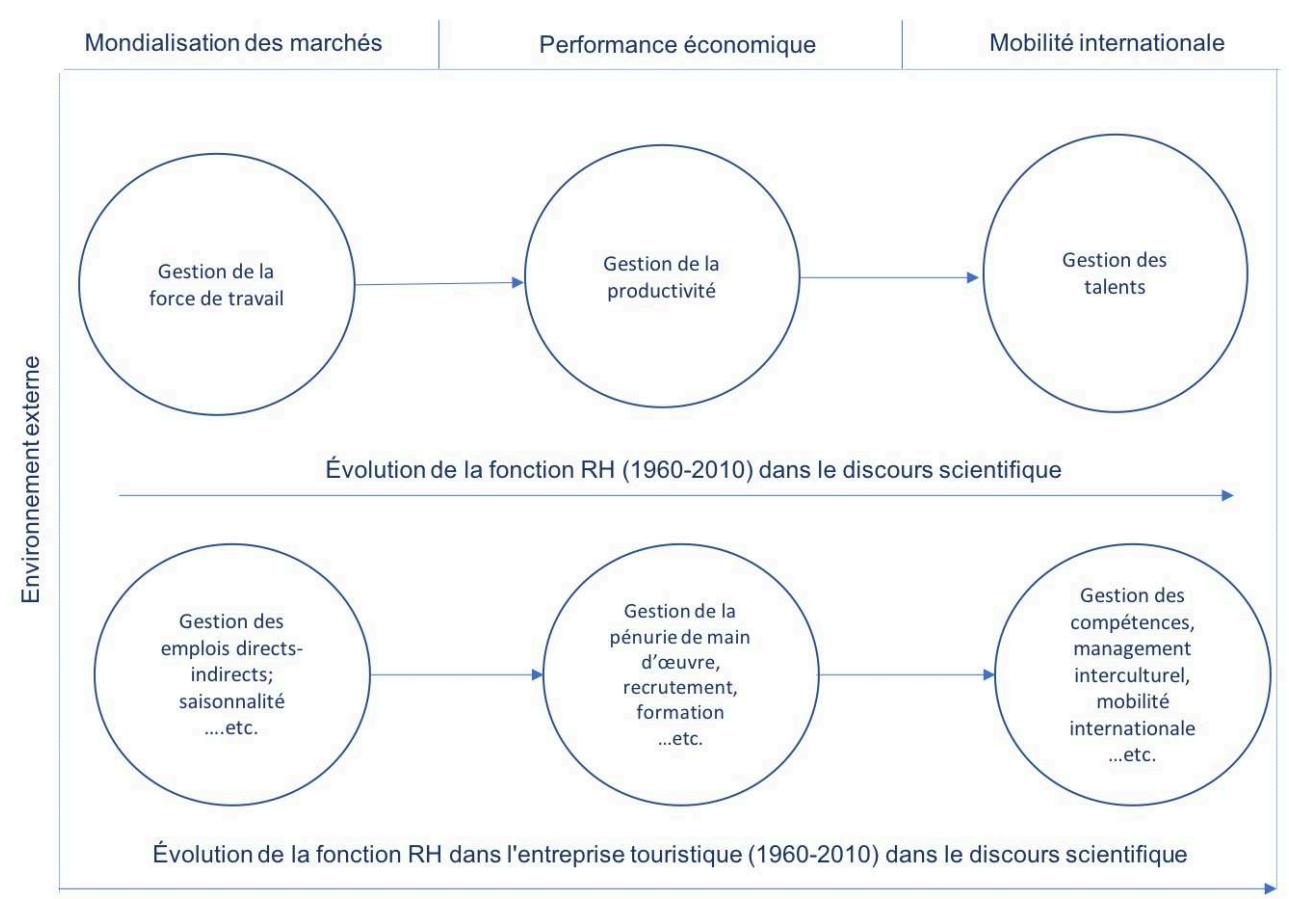

Source : les auteurs

\section{Méthodologie}

La perspective que nous proposons est une interrogation de type épistémologique concernant la construction du discours sur la gestion des ressources humaines par les spécialistes du tourisme. Nous privilégions une approche de type constructiviste pour l'analyse du discours des chercheurs. Nous appréhendons cette analyse de la gestion des ressources humaines en tourisme à travers les écrits parus dans la revue francophone Espaces tourisme \& loisirs. Non seulement celle-ci est une importante revue sur le plan du discours professionnel, mais elle permet d'apporter un regard diachronique sur l'évolution du discours en gestion des ressources humaines et ce, depuis les années 1990.

Nous faisons plus spécifiquement appel à deux types d'analyse :

-D'une part, l'analyse thématique afin de faire émerger des «thèmes représentatifs du contenu analysé, et ce, en rapport avec l'orientation de recherche (problématique)». Cette méthode a deux fonctions : faire émerger des thèmes pertinents d'un corpus et ensuite rechercher des récurrences et des regroupements (Paillé et Muchielli, 2008). Notre grille d'analyse a été constituée en privilégiant le mode de "thématisation séquenciée » : tirer un échantillon de textes au hasard, les analyser pour en constituer une fiche thématique. Si la fiche est retenue, elle est utilisée pour les autres textes. Cette démarche est appliquée quand le corpus est dense et qu'il est possible de travailler en équipe. Un exemple de cette grille, confectionnée pour les besoins de notre analyse thématique, est présenté dans la partie 3 ( $c f$. tableau 1). Nous avons utilisé cette grille pour l'analyse des 63 textes retenus. 
- D'autre part, l'analyse des "catégories conceptualisantes", qui permet, selon Paillé et Muchielli (2008, p. 233), de dépasser la simple analyse thématique du discours et d'apporter les premiers éléments de théorisation d'un phénomène. Nous appliquons cette méthode (que ces deux auteurs ont utilisée dans le cadre d'expériences et d'entreprises en psychologie) à notre matériau, constitué par les observations et les pratiques liées à la gestion des ressources humaines en tourisme et relevées dans les écrits publiés dans la revue Espaces tourisme \& loisirs. Le travail d'analyse consiste, selon Paillé et Muchielli, à construire une catégorie qui mènera vers la théorisation. La catégorie est constituée au fur et à mesure de l'analyse d'une interview. Pour notre travail d'analyse, il s'agissait des thèmes et des sousthèmes d'analyse en ressources humaines, issus des écrits de la revue Espaces tourisme \& loisirs. La catégorie est définie par ces deux mêmes auteurs (2012, p. 234) comme désignant « directement un phénomène. Elle représente la pratique par excellence à travers laquelle se déploie l'analyse en acte. À la différence de la "rubrique" ou du "thème", elle va bien au-delà de la désignation de contenu pour incarner l'attribution même de la signification ».

En soulignant la relation avec la théorisation ancrée, Paillé et Muchielli estiment nécessaire de soulever des questions d'ordre épistémologique : "la catégorie est-elle une variable? Quelle relation avec le concept ? " Pour eux, la catégorie désigne un phénomène, le décrit et lui donne sens, montrant ainsi une perspective de construction conceptuelle et de théorisation : «elle [la catégorie] a les propriétés synthétique, dénominative et explicative d'un concept.», ajoutant que «[...] tout construit théorique tentant de mieux cerner l'expérience humaine est d'abord, à l'origine, une catégorie " (Paillé et Muchielli, 2008, p. 236-237). Aussi, suivant cette méthode, la catégorie est construite selon deux conditions : elle doit répondre, d'une part, à un travail de description analytique et, d'autre part, à un travail d'interprétation.

17 Notre corpus d'analyse est constitué des articles recensés dans la revue à partir de l'identifiant « ressources humaines » qui nous renvoie à 76 articles, dont certains n'étaient plus disponibles sur le site. À noter que nous n'avons pas retenu les articles constitués essentiellement d'entretiens, car non pertinents pour une analyse thématique. Ainsi, nous avons retenu 63 articles aux fins de l'analyse du discours.

\section{Analyse thématique du discours de la GRH : la revue Espaces tourisme \& loisirs}

18 Parmi les différentes techniques d'analyse de contenu, nous avons privilégié l'analyse thématique. Cette dernière consiste à déterminer à partir d'un corpus donné un certain nombre de thèmes représentatifs du contenu analysé, en rapport avec l'orientation de la recherche (la problématique). Pour effectuer cette recherche, rappelons que nous avons principalement utilisé un corpus de textes provenant de la revue francophone Espaces tourisme \& loisirs, corpus composé de 63 textes, parus sur trois périodes: années 1990, années 2000 et années 2010.

L'analyse thématique du discours de la GRH à partir du corpus de textes a nécessité deux étapes :

- La création d'un tableau de 5 colonnes: 1) textes; 2) thèmes; 3) mots et expressions significatifs relevés ; 4) relations du discours avec l'entreprise, la société, les institutions ; 5) relations du discours avec des théories (management, sociologie, etc.). En outre, les textes sont séparés selon les décennies : années 1990-1999 (3 textes); années 2000-2009 (43 textes); 
années 2010-2015 (17 textes); cela permet de construire l'information en vue de son utilisation pour les différentes analyses qui suivront tout au long du texte.

- L'identification des thèmes et des axes thématiques des 63 textes, qui sont rassemblés respectivement dans les tableaux 2 et 3.

Tableau 1. Exemple de grille d'analyse devant servir à l'analyse thématique et à l'analyse par catégories « conceptualisantes »

\begin{tabular}{|c|c|c|c|c|c|}
\hline \multicolumn{6}{|c|}{ Décennie 1990} \\
\hline Textes & Thèmes & $\begin{array}{l}\text { Mots et } \\
\text { expressions } \\
\text { significatifs } \\
\text { relevés }\end{array}$ & $\begin{array}{l}\text { Relations du } \\
\text { discours avec } \\
\text { l'entreprise, } \\
\text { la société, les } \\
\text { institutions }\end{array}$ & $\begin{array}{l}\text { Relations du } \\
\text { discours avec } \\
\text { des théories } \\
\text { (management, } \\
\text { sociologie, etc.) }\end{array}$ & $\begin{array}{l}\text { Expression } \\
\text { pré- } \\
\text { catégorielle }\end{array}$ \\
\hline $\begin{array}{l}\text { "Qualité: } \\
\text { L'expérience } \\
\text { du groupe } \\
\text { Accor» } \\
\text { Chantal } \\
\text { Haas } \\
1994 \\
\text { Revue } \\
\text { Espaces } \\
\text { tourisme \& } \\
\text { loisirs, } \mathrm{n}^{\circ} \\
125\end{array}$ & $\begin{array}{l}\text { L'interdépendance } \\
\text { entre la satisfaction } \\
\text { client et la } \\
\text { satisfaction des } \\
\text { employés } \\
\text { Les attentes de la } \\
\text { clientèle dans le } \\
\text { milieu hôtelier }\end{array}$ & $\begin{array}{l}\text { Les relations } \\
\text { employeur/ } \\
\text { employé } \\
\text { Les exigences } \\
\text { des clients } \\
\text { Les attentes } \\
\text { des clients } \\
\text { La formation } \\
\text { des employés }\end{array}$ & $\begin{array}{l}\text { Culture } \\
\text { d'entreprise } \\
\text { internationale } \\
\text { Accor }\end{array}$ & $\begin{array}{l}\text { Gestion } \\
\text { ressources } \\
\text { humaines }\end{array}$ & $\begin{array}{l}\text { Adaptation } \\
\text { contrainte }\end{array}$ \\
\hline
\end{tabular}

Source : les auteurs

L'analyse thématique a été réalisée en trois phases: une analyse à partir du logiciel NVivo, une analyse des axes thématiques et une analyse des caractéristiques du discours en rapport avec le processus scientifique.

\subsection{L'analyse à partir du logiciel NVivo}

Cette première analyse de contenu, très sommaire, se fonde sur deux aspects : la récurrence des mots et un regroupement selon un diagramme. Dans cette analyse, deux principaux regroupements d'occurrences ressortent très nettement parmi les thèmes analysés et présentent une interrelation très étroite. Le premier regroupement rassemble les mots " communication", "web", sociaux, "réseaux ", « recrutement", «travail » et « employeur ». L'utilisation des réseaux sociaux et d'Internet transforme les pratiques du recrutement mais aussi les stratégies managériales relatives aux modes de visibilité et de recherche de compétences. Le deuxième regroupement est constitué de plusieurs termes très liés: "mobilité ", "professionnelle », " emplois ", " compétences", «formation ", " ressource ", " gestion", « culture ", " tourisme ", «touristique », « jeunes », « entreprise », «GRH», « développer ». L'emploi dans les secteurs touristique et hôtelier ayant pour caractéristiques d'être saisonnier, précaire 
et soumis à une mobilité importante, il impose des défis en termes de recrutement et d'attractivité de main-d'œuvre, particulièrement pour les jeunes, et l'entreprise tente de s'y adapter à travers l'utilisation des réseaux sociaux et des nouvelles technologies, une meilleure identification de sa culture d'entreprise, la valorisation des qualifications par la formation, tout en édifiant la mobilité dans le milieu touristique et hôtelier comme un moyen d'acquisition des compétences. De ce fait, tendre vers une gestion des ressources humaines qui évolue en permanence et s'adapte en conséquence devient une exigence constante pour l'entreprise.

Tableau 2. Fréquence des mots (généré par le logiciel Nvivo, 2019)

\begin{tabular}{|l|l|l|l|}
\hline Mot & Récurrence & Mot & Récurrence \\
\hline recrutement & 19 & professionnelle & 9 \\
\hline tourisme & 18 & sociaux & 9 \\
\hline entreprise & 15 & culture & 8 \\
\hline mobilité & 13 & développer & 8 \\
\hline touristique & 13 & emploi & 8 \\
\hline travail & 13 & jeunes & 8 \\
\hline compétences & 12 & ressources & 8 \\
\hline gestion & 12 & web & 8 \\
\hline GRH & 12 & réseaux & 7 \\
\hline formation & 10 & communication & 6 \\
\hline & & employeur & 6 \\
\hline
\end{tabular}


Figure 2. Diagramme des fréquences et regroupements des mots

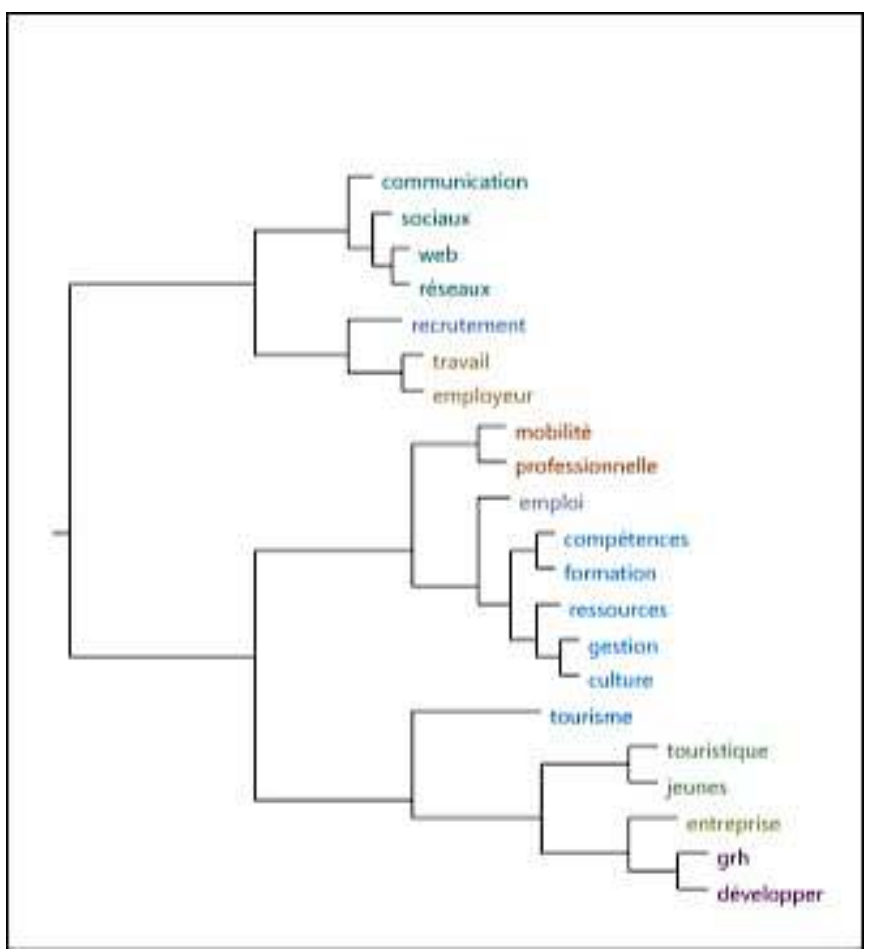

Source : généré par le logiciel NVivo, 2019

\subsection{L'analyse des regroupements et des axes thématiques}

Pour l'analyse thématique, nous avons d'abord procédé à l'identification du regroupement des thèmes des 63 textes ( $c f$. tableau 3), permettant d'engager la procédure de récurrence (thèmes répétitifs) à partir de cinq axes thématiques. Le tableau 4 montre l'organisation des cinq axes thématiques, auxquels se rattachent les thèmes identifiés dans le tableau 3 en fonction de la décennie et de leur ordre numérique.

Tableau 3. Identification des thèmes par décennie

\begin{tabular}{|l|l|l|}
\hline 1990-1999 & $\begin{array}{l}\text { 24-Conditions de travail } \\
\text { difficiles }\end{array}$ & 2010-2015 \\
\hline $\begin{array}{l}\text { 1-Interdépendance de la } \\
\text { satisfaction client-employé }\end{array}$ & $\begin{array}{l}\text { 25-Pratiques de recrutement } \\
\text { difficiles }\end{array}$ & $\begin{array}{l}\text { 47-Assurer la réputation de } \\
\text { l'entreprise par le web }\end{array}$ \\
\hline $\begin{array}{l}\text { 2-Recherche d'un } \\
\text { fonctionnement managérial } \\
\text { adapté }\end{array}$ & $\begin{array}{l}\text { 26-Inadaptation } \\
\text { organismes territoriaux en } \\
\text { tourisme }\end{array}$ & $\begin{array}{l}\text { 48-Importance du web et de sa } \\
\text { gestion }\end{array}$ \\
\hline $\begin{array}{l}\text { 3-Harmonisation du dialogue } \\
\text { entre acteurs de l'entreprise }\end{array}$ & $\begin{array}{l}\text { 27-Redécouverte des valeurs } \\
\text { et de la culture d'entreprise }\end{array}$ & $\begin{array}{l}\text { 49-Réseaux sociaux comme outil } \\
\text { de communication }\end{array}$ \\
\hline
\end{tabular}




\begin{tabular}{|c|c|c|}
\hline 2000-2009 & $\begin{array}{l}\text { 28-Précarité de l'emploi } \\
\text { comme obstacle au } \\
\text { fonctionnement }\end{array}$ & $\begin{array}{l}\text { 50-Visibilité de l'entreprise par } \\
\text { les réseaux sociaux comme } \\
\text { Facebook }\end{array}$ \\
\hline $\begin{array}{l}\text { 4-Diversité d'emploi et culture } \\
\text { d'entreprise }\end{array}$ & $\begin{array}{l}\text { 29-Reconnaissnce des acquis } \\
\text { professionnels }\end{array}$ & $\begin{array}{l}\text { 51-Transformation des pratiques } \\
\text { de gestion par le web }\end{array}$ \\
\hline $\begin{array}{l}\text { 5-Réalités touristiques et } \\
\text { adaptation des organismes }\end{array}$ & $\begin{array}{l}\text { 30-Considérer la culture } \\
\text { d'entreprise internationale }\end{array}$ & $\begin{array}{l}\text { 52-Usage limité du web par les } \\
\text { organismes }\end{array}$ \\
\hline $\begin{array}{l}\text { 6-Utilisation de la fanfare pour } \\
\text { construire la relation humaine }\end{array}$ & $\begin{array}{l}\text { 31-Importance de la GRH } \\
\text { comme fonction vitale }\end{array}$ & $\begin{array}{l}\text { 53-Usage limité du web par les } \\
\text { organismes }\end{array}$ \\
\hline $\begin{array}{l}\text { 7- Précarité de l'emploi chez les } \\
\text { jeunes }\end{array}$ & $\begin{array}{l}\text { 32- Facteur humain comme } \\
\text { fondamental en tourisme }\end{array}$ & $\begin{array}{l}\text { 54-Recrutement par les } \\
\text { organismes-conseils }\end{array}$ \\
\hline $\begin{array}{l}\text { 8-Formation comme vecteur de } \\
\text { formation de la RH }\end{array}$ & $\begin{array}{l}\text { 33-Nécessité des profils } \\
\text { diversifiés }\end{array}$ & $\begin{array}{l}\text { 55-Jeunes face au changement } \\
\text { dans l'organisation du travail }\end{array}$ \\
\hline $\begin{array}{l}\text { 9- GRE pour contrer la } \\
\text { saisonnalité }\end{array}$ & $\begin{array}{l}\text { 34-Importance des tests et } \\
\text { mise en situation }\end{array}$ & $\begin{array}{l}\text { 56-Perception du travail par les } \\
\text { jeunes salariés }\end{array}$ \\
\hline $\begin{array}{l}\text { 10-Inégalité homme-femme dans } \\
\text { l'emploi }\end{array}$ & $\begin{array}{l}\text { 35-Ressource humaine en } \\
\text { évolution constante }\end{array}$ & $\begin{array}{l}\text { 57-Contrainte de l'emploi } \\
\text { atypique }\end{array}$ \\
\hline 11-Repenser le modèle de la DRH & $\begin{array}{l}\text { 36-Assurer le développement } \\
\text { personnel par le coaching }\end{array}$ & $\begin{array}{l}\text { 58-Contrainte de situation } \\
\text { saisonnalité-fidélisation }\end{array}$ \\
\hline $\begin{array}{l}\text { 12-Repenser les compétences du } \\
\text { manager }\end{array}$ & $\begin{array}{l}\text { 37-Mobilité internationale } \\
\text { comme atout de la GRH }\end{array}$ & $\begin{array}{l}\text { 59-Groupement employeur (GE) } \\
\text { comme outil de la GRH }\end{array}$ \\
\hline 13-Difficultés de recrutement & $\begin{array}{l}\text { 38-Communauté de salariés } \\
\text { comme valeur }\end{array}$ & $\begin{array}{l}\text { 60- Groupement employeur (GE) } \\
\text { comme outil de la GRH }\end{array}$ \\
\hline $\begin{array}{l}\text { 14-Prestation de service en } \\
\text { hôtellerie-restauration }\end{array}$ & $\begin{array}{l}\text { 39-Identification du potentiel } \\
\text { à la mobilité }\end{array}$ & $\begin{array}{l}\text { 61- Groupement employeur (GE) } \\
\text { comme outil de management de } \\
\text { la RH }\end{array}$ \\
\hline $\begin{array}{l}\text { 15-Nouvelles technologies } \\
\text { comme lien entre tradition et } \\
\text { modernité }\end{array}$ & $\begin{array}{l}\text { 40-Formation de la ressource } \\
\text { humaine }\end{array}$ & $\begin{array}{l}\text { 62-Conception de la mobilité } \\
\text { comme atout }\end{array}$ \\
\hline $\begin{array}{l}\text { 16-Transformation du métier } \\
\text { par les nouvelles technologies }\end{array}$ & $\begin{array}{l}\text { 41-Le saisonnier face à la } \\
\text { règlementation }\end{array}$ & $\begin{array}{l}\text { 63-Concevoir la mobilité comme } \\
\text { atout }\end{array}$ \\
\hline $\begin{array}{l}\text { 17-Transformations } \\
\text { management et de la GRH }\end{array}$ & $\begin{array}{l}\text { 42-Reconnaissance des } \\
\text { communautés salariés-clients }\end{array}$ & \\
\hline 18-Les jeunes face à l'emploi & $\begin{array}{l}\text { 43-Importance } \\
\text { l'interculturel en GRH }\end{array}$ & \\
\hline
\end{tabular}




\begin{tabular}{|l|l|}
\hline $\begin{array}{l}\text { 19-Innovation dans l'emploi } \\
\text { comme moyen d'insertion des } \\
\text { jeunes }\end{array}$ & $\begin{array}{l}\text { 44-Identification des besoins } \\
\text { internes par l'audit externe }\end{array}$ \\
\hline $\begin{array}{l}\text { 20-Attentes du salarié comme } \\
\text { facteur de recrutement }\end{array}$ & $\begin{array}{l}45 \text { Évolution constante des } \\
\text { métiers }\end{array}$ \\
\hline $\begin{array}{l}\text { 21-Adaptation de la ressource } \\
\text { humaine en hôtellerie-tourisme }\end{array}$ & $\begin{array}{l}\text { 46-Soutien à l'analyse des } \\
\text { compétences }\end{array}$ \\
\cline { 1 - 2 } $\begin{array}{l}\text { 22-Difficultés de recrutement } \\
\text { des jeunes en tourisme- } \\
\text { hôtellerie }\end{array}$ & \\
\hline $\begin{array}{l}\text { 23-Difficultés du management } \\
\text { en situation de crise économique }\end{array}$ & \\
\hline
\end{tabular}

Parmi les thèmes regroupés autour des cinq axes thématiques ( $c f$. tableau 4 ), on peut observer que les axes thématiques $2,3,4$ et 5 représentent $93,5 \%$ de la réalité thématique organisée à partir des 63 textes analysés. Toutefois, cette réalité est formée d'une situation double exprimant deux pôles du discours professionnel sur la GRH, se présentant en forme de « double contrainte » :

- une réalité interne, formée de contraintes, organisée autour de «la problématique de l'emploi » (axe 4), regroupant 26,9\% des thématiques du discours professionnel ;

- une réalité externe (l'environnement), représentée par l'axe 2 (Nouvelles technologies) et l'axe 3 (Repenser les modèles et pratiques); cette réalité exprime l'exigence d'adaptation (insertion des technologies, modèles managériaux repensés) à une situation de contraintes représentée par la réalité interne (Problématique de l'emploi).

Tableau 4. Axes thématiques et récurrences des thèmes relatifs aux 63 textes

\begin{tabular}{|c|c|c|c|c|}
\hline $\begin{array}{l}\text { Périodes } \\
\text { Axes thématiques }\end{array}$ & 1990-1999 & 2000-2009 & 2010-2015 & Total \\
\hline 1-La recherche d'une culture d'entreprise & - & 4 & - & $\begin{array}{l}4 \\
(6,3 \%)\end{array}$ \\
\hline $\begin{array}{l}\text { 2-Nouvelles technologies et transformation de la } \\
\text { gestion }\end{array}$ & - & 2 & 7 & $\begin{array}{l}9 \\
(14.3 \%)\end{array}$ \\
\hline $\begin{array}{l}\text { 3-Repenser les modèles et pratiques } \\
\text { (management, GRH, DRH) }\end{array}$ & - & 6 & 7 & $\begin{array}{l}13 \\
(20,6 \%)\end{array}$ \\
\hline $\begin{array}{l}\text { 4-Problématique de l'emploi (précarité, } \\
\text { formation, saisonnalité, inégalité, recrutement) }\end{array}$ & - & 14 & 3 & $\begin{array}{l}17 \\
(26,9 \%)\end{array}$ \\
\hline $\begin{array}{l}\text { 5-Adaptation aux réalités et contraintes de } \\
\text { l'entreprise }\end{array}$ & 3 & 17 & - & $\begin{array}{l}20 \\
(31,7 \%)\end{array}$ \\
\hline
\end{tabular}




\begin{tabular}{|l|l|l|l|l|}
\hline Total textes & 3 textes & 43 textes & 17 textes & $\begin{array}{l}63 \\
\text { textes }\end{array}$ \\
\hline
\end{tabular}

Source : les auteurs

La nécessité d'une adaptation (cf. figure 3), qui représente également une contrainte et une exigence (axe 5 : «Adaptation aux réalités et contraintes de l'entreprise »), relie les deux réalités (interne et externe) observées plus haut ( $c f$. tableau 4) et montre aussi qu'il y a une difficulté à la mettre en œuvre : par exemple, la « Recherche d'une culture d'entreprise » (axe 1) ne représente que 6,3\% de l'analyse thématique.

Figure 3

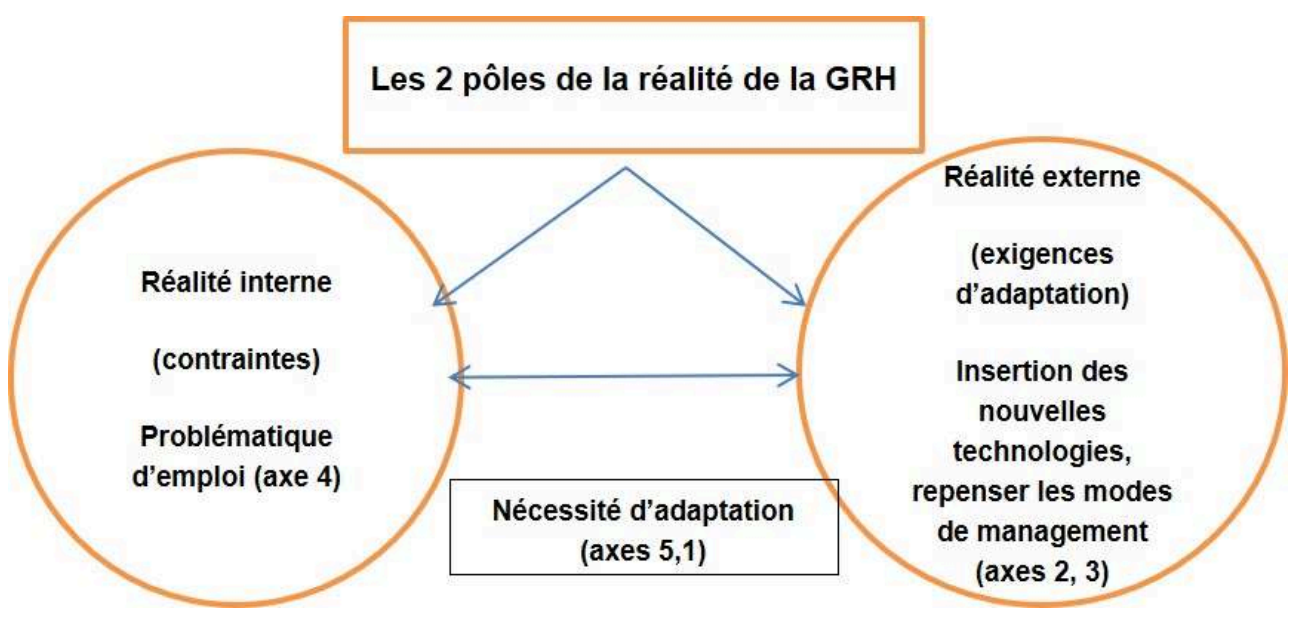

Ainsi, cette première identification de la réalité thématique (63 textes) montre bien une situation en forme de "double contrainte »: d'une part, les conditions d'emploi et d'organisation du travail ont un poids important et, d'autre part, la nécessité d'adaptation à cette réalité (condition interne) et aux exigences des environnements (condition externe). S'agissant de ce dernier aspect, il s'agit aussi de la nécessité de repenser le travail et l'organisation imposée par le renouvellement du management, l'évolution de la société et l'impact des TI (technologies de l'information), qui imposent à leur tour des changements et deviennent ainsi une exigence d'adaptation. Enfin, en regroupant les axes 2, 3 et 5, l'importance de la contrainte d'adaptation de l'entreprise et des salariés s'accroît davantage, totalisant plus des deux tiers $(66,6 \%)$ des thèmes retenus dans les 63 textes.

L'image proposée à travers ces deux pôles - une "réalité interne » (contraintes de la situation d'emploi) et une "réalité externe " (exigences d'adaptation) - nous montre deux caractéristiques : une grande part (2/3) est accordée à la contrainte d'adaptation (axes 2, 3, 5 et 1 ) et une faible part (1/3) revient à la problématique de l'emploi - sans que cela ne l'amoindrisse pour autant. En effet, nous faisons face à une situation complexe : comment concilier les deux aspects de cette dernière, organisée autour d'une situation contradictoire qui nécessite d'évoluer avec les conditions diverses de l'activité d'emploi en tourisme? Faut-il reconsidérer le modèle de management du tourisme davantage pris dans un processus de recherche de solutions à des problèmes, d'où l'importance plus grande accordée aux pratiques et moins à l'analyse de type conceptuel? 


\subsection{Les caractéristiques du discours en rapport avec le processus scientifique}

27 Nous avons par ailleurs recherché certains aspects spécifiques dans le but d'observer le lien du discours sur la GRH avec la perspective scientifique. Nous avons privilégié pour cette analyse les années 2009 ( 17 textes), 2013 ( 8 textes) et 2015 (9 textes), le tout rassemblant 34 textes, soit $54 \%$ de l'ensemble des textes analysés ( $c f$. tableau 5 ).

Tableau 5. Le discours sur la GRH en tourisme, à travers quelques indicateurs

\begin{tabular}{|c|c|c|c|}
\hline $\begin{array}{l}\text { Années } \\
\text { Indicateurs }\end{array}$ & 2009 & 2013 & 2015 \\
\hline $\begin{array}{l}\text { Pratiques/ } \\
\text { expériences }\end{array}$ & $\begin{array}{l}10 \text { entreprises dont } \\
4 \text { grandes } \\
2 \text { grands hôtels }\end{array}$ & $\begin{array}{l}7 \text { entreprises dont } \\
3 \text { grandes } \\
1 \text { organisme }\end{array}$ & $\begin{array}{l}6 \text { entreprises et } \\
3 \text { organismes publics }\end{array}$ \\
\hline Méthodes & 5 & 8 & 6 \\
\hline $\begin{array}{l}\text { Approches/ } \\
\text { théories }\end{array}$ & 2 & - & 3 \\
\hline $\begin{array}{l}\text { Acteurs } \\
\text { (analystes) }\end{array}$ & $\begin{array}{l}\text { Entreprise : } 6 \\
\text { Banque : } 1 \\
\text { Université : } 1 \\
\text { Cabinet : } 8 \\
\text { Organisme public : } 1\end{array}$ & $\begin{array}{l}\text { Entreprise : } 3 \\
\text { Cabinet : } 5\end{array}$ & $\begin{array}{l}\text { Entreprise : } 1 \\
\text { Université : } 2 \\
\text { Cabinet : } 5 \\
\text { Organisme public : } 1\end{array}$ \\
\hline Total textes & 17 textes & 8 textes & 9 textes \\
\hline
\end{tabular}

Source : les auteurs

Cette analyse de la relation entre le discours et sa base théorique ou pratique portait sur l'identification de certains indicateurs :

- pratiques et expériences: la réalité du discours en lien avec la pratique et l'expérience d'entreprise ;

- méthodes : le recours aux outils relevant du management d'entreprise ;

- approches : les analyses relevant des sciences humaines et sociales ;

- acteurs (DRH, consultant, universitaire) : statut des acteurs analysant la situation de la gestion des ressources humaines dans les entreprises.

L'analyse de la réalité du discours, pour la période 2009-2015, nous permet de relever les aspects suivants :

- L'intérêt des pratiques et expériences associées à l'entreprise sont reconnues (recrutement, formation, mobilité, etc.) durant cette période. Cette observation des pratiques et des expériences se voit aussi dans les grands hôtels (2) et les grandes entreprises, en moins grand nombre (7 cas), qui se présentent comme des acteurs importants dans la diffusion des 
procédés managériaux liés à la gestion des ressources humaines en période de changement continu.

- L'indicateur « outil, méthodes » (25/34) nous permet également d'observer un intérêt pour le développement d'instruments d'organisation en matière de GRH, d"analyse de l'emploi et de fonctionnement organisationnel, renforçant ainsi la nécessité d'adaptation à la contrainte, ainsi qu'une garantie de rationalisation.

-L'indicateur "approches, théories» (5/34) montre que la dimension scientifique d'une analyse de GRH reste encore peu développée. Toutefois, on note également une orientation vers la remise en question de certaines problématiques internes à l'activité touristique (les contraintes), telles la mobilité des salariés, l'analyse et l'organisation du travail, analysées par une sociologie industrielle et du travail jadis en vigueur dans un contexte de stabilité (années 1950-1980) et aujourd'hui bousculée dans ses concepts (Mercure et Vultur, 2019). Par ailleurs, la référence à des approches et des théories n'est pas seulement le fait de l'université, mais aussi de chercheurs autonomes ou dans des cabinets de conseil.

- L'analyse du discours sur la gestion des ressources humaines est engagée surtout par deux acteurs clés : les cabinets de conseil et les entreprises, représentant respectivement $53 \%$ et $29,5 \%$ de l'ensemble des 34 textes analysés. On retiendra ici que parmi les entreprises engagées, on retrouve des responsables de GRH ou DRH de grandes entreprises, telles que Disney, Accor, Club Med, ainsi que des hôtels de luxe.

\section{Analyse du discours de la GRH selon les catégories conceptualisantes}

Suite à l'analyse thématique, qui nous a permis de développer une première réflexion sur le discours en GRH à travers les articles de la revue Espaces tourisme \& loisirs, nous poursuivons par une analyse des catégories conceptualisantes. Cette dernière nous rapproche davantage du cœur conceptuel du discours et, par là même, nous permet d'affiner la construction des connaissances en ce sens. L'analyse des catégories conceptualisantes est effectuée selon les étapes suivantes :

1. l'élaboration d'expressions catégorielles primaires, à partir des thèmes et sous-thèmes : il s'agit ici de réduire les thèmes et sous-thèmes ainsi que les expressions utilisées en expressions que nous identifions comme catégorielles pour chaque texte ;

2. une catégorisation dite "catégories conceptualisantes secondaires" réalisée à partir des expressions catégorielles primaires;

3. la construction de catégories conceptualisantes principales.

31 D'après un premier regroupement des thèmes et sous-thèmes relevés dans chaque article, par décennie, on dénombrait 63 catégorisations primaires (les 63 articles retenus pour l'analyse), que nous avons regroupées en catégories conceptualisantes secondaires (cf. tableau 6).

Ainsi, durant la décennie 1990, seuls trois textes ont été identifiés, dont les expressions primaires ont été regroupées autour de la catégorie conceptualisante secondaire de " contexte managérial contraignant ». Cela désigne une adaptation visée et souhaitée, mais l'objectif d'adaptation est contraint du fait d'une situation difficile et complexe de l'activité touristique, une situation souvent rappelée dans les écrits de type professionnel autant que conceptuel. 
Tableau 6. Regroupement des expressions catégorielles primaires et des catégories conceptualisantes secondaires, élaborées à partir des 63 textes considérés comme matériau d'analyse du discours en ressources humaines, extraits de la revue Espaces tourisme \& loisirs (période 1999-2015)

\begin{tabular}{|c|c|}
\hline conceptualisantes & Expressions catégorielles primaires \\
\hline Contexte managérial contraignant & $\begin{array}{l}\text { DÉCENNIE 1990-1999-3 TEXTES } \\
\text { Contrainte d'exigence des clients / satisfaction des } \\
\text { employés } \\
\text { Nécessité d'une adaptation permanente } \\
\text { Contrainte de mobilité / nécessité de concertation }\end{array}$ \\
\hline DÉCENNIE 2000-2009 - 43 TEXTES & \\
\hline Adaptation de l'organisation & $\begin{array}{l}\text { Construire sa culture d'entreprise } \\
\text { L'adaptation par la concertation } \\
\text { Découvrir sa culture d'entreprise } \\
\text { Connaître la culture d'entreprise des groupes } \\
\text { internationaux } \\
\text { Hôtellerie entre tradition et innovation } \\
\text { Développer la compétence interculturelle }\end{array}$ \\
\hline Adaptation des ressources humaines & $\begin{array}{l}\text { Adapter les RH à l'organisation touristique } \\
\text { Repenser le rôle du DRH } \\
\text { Reconstruire la GRH } \\
\text { Revoir la GRH face au changement } \\
\text { Repenser la fonction GRH } \\
\text { Repenser la perception vis-à-vis du travail } \\
\text { La problématique du recrutement } \\
\text { Le recrutement à l'étude } \\
\text { La fonction GRH intégrée à l'activité } \\
\text { L'importance du facteur humain } \\
\text { L'importance des profils diversifiés } \\
\text { Reconnaître davantage les tests psychométriques } \\
\text { Adapter la GRH aux clients } \\
\text { La gestion du service en hôtellerie } \\
\text { Le projet de mobilité comme moteur de la GRH } \\
\text { Assurer l'intégration permanents-saisonniers } \\
\text { La connaissance des besoins réels par l'audit } \\
\text { La nécessité de l'analyse de l'emploi } \\
\text { La mobilité internationale comme opportunité } \\
\text { La construction personnelle par le coaching }\end{array}$ \\
\hline
\end{tabular}




\begin{tabular}{|c|c|}
\hline Conditions de travail difficiles & $\begin{array}{l}\text { La contrainte de la précarité de l'emploi } \\
\text { La contrainte de la saisonnalité professionnelle } \\
\text { Le problème de la discrimination hommes-femmes } \\
\text { L'équilibre difficile entre pénurie et fidélisation de la } \\
\text { main-d'œuvre } \\
\text { Les conditions d'emploi difficiles } \\
\text { Les conditions sociales difficiles des saisonniers } \\
\text { Le management inadapté des organisations territoriales } \\
\text { Penser les conditions sociales du travail } \\
\text { La reconnaissance des acquis professionnels } \\
\text { La communauté des salariés } \\
\text { L'inégalité des saisonniers face à la règlementation }\end{array}$ \\
\hline $\begin{array}{l}\text { Nécessité de la formation et } \\
\text { reconnaissance des qualifications }\end{array}$ & $\begin{array}{l}\text { La formation des personnes } \\
\text { Le passage du métier au service } \\
\text { L'emploi et les jeunes } \\
\text { La professionnalisation des jeunes } \\
\text { La garantie de la diplomation des salariés } \\
\text { La valorisation des métiers }\end{array}$ \\
\hline \multicolumn{2}{|c|}{ DÉCENNIE 2010 (JUSQU'À 2015) - 17 TEXTES } \\
\hline Adaptation par le web & $\begin{array}{l}\text { Renforcer la visibilité de l'entreprise par le web (2) } \\
\text { Renforcer le recrutement et la recherche de compétences } \\
\text { par l'utilisation des réseaux sociaux (3) }\end{array}$ \\
\hline La perception nouvelle du travail & $\begin{array}{l}\text { Nouvelles générations et perceptions nouvelles face au } \\
\text { temps de travail (2) } \\
\text { La mobilité comme opportunité (2) }\end{array}$ \\
\hline $\begin{array}{l}\text { La contrainte situationnelle de } \\
\text { l'activité }\end{array}$ & $\begin{array}{l}\text { Adaptation lente d'organismes et agences au recrutement } \\
\text { par le web (2) } \\
\text { Recrutement par les cabinets de conseil } \\
\text { La double contrainte d'adaptation flexibilité-sécurité } \\
\text { La double contrainte saisonnalité-fidélisation de la main- } \\
\text { d'œuvre } \\
\text { Difficile adaptation de l'outil "groupement } \\
\text { d'employeurs » à la situation touristique (3) }\end{array}$ \\
\hline
\end{tabular}

La décennie 2000-2009 est la plus fournie en textes (43) et l'on dénombre autant d'expressions catégorielles primaires reflétant le discours des auteurs. À la suite des 43 expressions catégorielles, quatre catégories conceptualisantes secondaires ont été identifiées, à partir de la problématique de l'adaptation (entreprise, organisation territoriale, individus): 1) adaptation de l'organisation (6) (culture d'entreprise, management organisationnel, etc.); 2) adaptation des RH à l'organisation (20) (perception de la mobilité, nécessité de repenser les RH et le DRH, etc.) ; 3) conditions 
de travail difficiles (11) (nouvelles perceptions du travail, conditions d'emploi, etc.); et 4) nécessité de la formation et reconnaissance des qualifications (6) (adaptation des métiers et des personnes). Les catégories 2 et 3 sont les plus fournies $(20,11)$, soit plus de $72,1 \%$ des catégories de la décennie et $49,2 \%$ de l'ensemble des catégories analysées (63). Dans la catégorie 2 , « la mobilité professionnelle » est considérée comme une opportunité (mobilité internationale comme projet) ou selon une perspective de nouvelle perception du travail, alors qu'elle était perçue comme une contrainte dans les années 1990.

Ce qui ressort des quatre catégories de la décennie 2000-2009, c'est la nécessite de passer d'une situation qui génère une contrainte inhérente à l'activité touristique à une perspective consistant à développer une nouvelle perception du travail, des conditions de mobilité, et à adapter les outils d'organisation à la situation particulière des RH en tourisme.

Le discours de la GRH en tourisme évolue donc selon une constante forte et contrainte, celle de la situation touristique: dans la décennie 1990 (réduite toutefois à trois articles), on s'oriente vers l'observation de l'existence d'une situation complexe, alors que la décennie 2000-2009 voit la nécessité d'une perception nouvelle de l'adaptation face à une situation touristique encore alourdie de contraintes diverses (précarité, inégalité dans l'emploi, notamment des saisonniers face à la règlementation sociale, mobilité, etc.).

Dans la décennie 2010 (jusqu'en 2015), le discours évolue dans le même sens que la décennie précédente, avec en plus la nécessité d'une adaptation aux technologies numériques.

En effet, au cours des années 2010-2015, on retrouve le même noyau central du discours, à savoir la situation complexe dans laquelle évolue l'activité touristique, avec toutefois l'intégration d'une nouvelle perception à travers, notamment, deux aspects : d'une part, l'opportunité offerte par le web (réseaux professionnels), permettant d'ouvrir de nouvelles possibilités à l'entreprise touristique (marque, visibilité, élargissement du bassin des compétences) et, d'autre part, l'appréhension d'une nouvelle situation (perception renouvelée du travail). Néanmoins, la situation complexe et permanente dans laquelle évolue l'activité touristique (précarité, saisonnalité, mobilité, etc.) demeure vivace.

Au cours de la période 2010-2015, on observe 17 expressions catégorielles primaires, que nous regroupons en trois catégories conceptualisantes secondaires : 1) l'adaptation par le web (5) ; 2) la perception nouvelle du travail (4); et 3) la contrainte situationnelle de l'activité (8). Les regroupements 1 et 2 , qui représentent $53 \%$ des expressions catégorielles primaires, peuvent être identifiés comme les deux catégories principales d'un premier noyau, celui de la perspective d'une adaptation aux changements divers (mondialisation, mobilité, travail, etc.). Le regroupement 3, autour de la catégorie "contrainte situationnelle de l'activité ", représentant $47 \%$ des expressions catégorielles, constitue le second noyau irréductible de l'activité (précarité, double contrainte, flexibilité, saisonnalité, etc.).

39 Finalement, cette analyse nous permet d'observer que le discours sur la gestion des ressources humaines en tourisme, à partir des écrits parus dans la revue Espaces tourisme \& loisirs, peut être compris comme étant formé d'une catégorie conceptualisante principale, formée de deux catégories conceptualisantes et identifiées 
comme étant ses deux noyaux opposés mais complémentaires. La catégorie conceptualisante principale est identifiée par « la double contrainte adaptative » et les deux noyaux qui la composent sont «la perception adaptative renouvelée » et «la contrainte situationnelle fonctionnelle » (cf. tableau 7).

Selon Paillé et Mucchielli (2012), la catégorie conceptualisante est caractérisée selon trois moments : la définition (ce qui désigne le phénomène comme arguments); les propriétés (ce qui distingue et caractérise le phénomène); et les conditions d'existence (les situations et les événements qui donnent vie au phénomène).

Tableau 7. Les catégories conceptualisantes issues du discours de la GRH en tourisme, à partir d'articles de la revue Espaces tourisme \& loisirs (1999-2015)

\begin{tabular}{|c|c|c|c|}
\hline $\begin{array}{l}\text { CATÉGORIES } \\
\text { CONCEPTUALISANTES }\end{array}$ & DÉFINITION & PROPRIÉTÉS & $\begin{array}{l}\text { CONDITIONS } \\
\text { D'EXISTENCE }\end{array}$ \\
\hline $\begin{array}{l}\text { La double contrainte } \\
\text { adaptative }\end{array}$ & $\begin{array}{ll} & \\
& \\
& \\
& \\
\text { «La double contrainte* } & \\
\text { se réfère à une } & \text { situation } \\
\text { aux } & \text { aspects } \\
\text { contradictoires, } & \text { rendant } \\
\text { difficiles } & \text { le } \\
\text { fonctionnement } & \text { et } \\
\text { l'adaptation } & \text { au } \\
\text { changement. } & \end{array}$ & $\begin{array}{l}\text { Mobilité } \\
\text { professionnelle } \\
\text { inhérente / } \\
\text { programme de } \\
\text { mobilité proposée } \\
\text { Saisonnalité } \\
\text { permanente / } \\
\text { meilleure } \\
\text { intégration } \\
\text { saisonniers- } \\
\text { permanents } \\
\text { Flexibilité / } \\
\text { sécurité } \\
\text { l'emploi dans } \\
\text { Saisonnalité / } \\
\text { fidélisation de la } \\
\text { main-d'œuvre }\end{array}$ & $\begin{array}{l}\text { Contexte marqué par } \\
\text { divers aspects } \\
\text { (traditions/ } \\
\text { innovations) } \\
\text { Activités de plus en } \\
\text { plus mondialisées / } \\
\text { perception négative } \\
\text { localement } \\
\text { Sensibilité de l'activité } \\
\text { à tout incident, mais } \\
\text { grande résilience }\end{array}$ \\
\hline $\begin{array}{l}\text { La contrainte } \\
\text { situationnelle } \\
\text { fonctionnelle }\end{array}$ & $\begin{array}{l}\text { Il s'agit ici des différents } \\
\text { aspects qui constituent la } \\
\text { situation fonctionnelle du } \\
\text { tourisme, à laquelle font } \\
\text { référence de nombreux } \\
\text { ouvrages et qui est } \\
\text { reconnue comme centrale. }\end{array}$ & $\begin{array}{l}\text { Précarité } \\
\text { l'emploi } \\
\text { Recrutement } \\
\text { difficile } \\
\text { Mobilité } \\
\text { professionnelle }\end{array}$ & $\begin{array}{l}\text { Situation de précarité, } \\
\text { mais nécessité } \\
\text { d'adaptation aux aléas } \\
\text { et aux clients } \\
\text { Inégalité } \\
\text { socioprofessionnelle } \\
\text { entre travailleurs } \\
\text { Adaptation lente de } \\
\text { l'organisation } \\
\text { Mondialisation } \\
\text { économique et sociale } \\
\text { plus prégnante }\end{array}$ \\
\hline
\end{tabular}




\begin{tabular}{|c|c|c|c|}
\hline $\begin{array}{l}\text { La perception } \\
\text { adaptative renouvelée }\end{array}$ & $\begin{array}{l}\text { Nouvelle vision de la façon } \\
\text { d'entrevoir les modes } \\
\text { d'organisation et la } \\
\text { situation de l'activité }\end{array}$ & $\begin{array}{l}\text { Accroissement de } \\
\text { l'utilisation des } \\
\text { réseaux sociaux } \\
\text { professionnels } \\
\text { Utilisation du web } \\
\text { pour la visibilité } \\
\text { entrepreneuriale } \\
\begin{array}{ll}\text { Adaptation des } \\
\text { nouvelles } \\
\text { générations aux } \\
\text { réalités de la } \\
\text { mondialisation }\end{array}\end{array}$ & $\begin{array}{l}\text { Besoins chez l'individu } \\
\text { et l'entreprise de } \\
\text { s'adapter au } \\
\text { changement interne et } \\
\text { international } \\
\text { Besoins de développer } \\
\text { des compétences } \\
\text { nouvelles } \\
\text { (interculturelles } \\
\text { internationales, } \\
\text { internes) }\end{array}$ \\
\hline
\end{tabular}

* en référence à la théorie de la double contrainte (double bind) de Gregory BATEson et al. $(1956,1980)$

41 La théorie de la «double contrainte » (double bind) de Bateson $(1956,1980)$ mobilisée dans notre recherche peut nous aider à comprendre en grande partie la réalité de l'activité touristique (entreprise, organisme) qui émerge à travers les discours des praticiens (entreprise, organisme, cabinet-conseil, consultant). Cette théorie, élaborée par Gregory Bateson, Don D. Jackson, Jay Haley et John Weakland en 1956, est basée sur l'observation de la communication du sujet avec sa famille. Cette relation s'engage à partir d'«injonctions paradoxales», dans lesquelles le sujet qui reçoit le message se trouve devant des énoncés logiques mais contradictoires et s'il réagit ou n'agit pas correctement, il risque d'être mal perçu (Watlzlawick et al., 1972).

Pour Watzlawick, Helmick et Jackson (1972), la situation de la double contrainte, dans la relation de communication, se présente en trois phases, selon lesquelles le message est ainsi structuré :

a) il [le message] affirme quelque chose; b) il affirme quelque chose sur sa propre affirmation; c) ces deux affirmations s'excluent. Ainsi, si le message est une injonction, il faut lui désobéir pour lui obéir... Le sens du message est donc indécidable...

Cette théorisation de la relation de communication développée dans le contexte de la psychiatrie-psychanalyse trouve aussi un intérêt dans le domaine des sciences humaines et sociales, notamment au niveau de l'entreprise (coaching, développement personnel, analyses des enjeux, etc.). Des auteurs y ont recours également pour observer la relation de l'organisation et le modèle de management (relations des individus avec les structures), comme Duterme (2008) et Casteigts (2017), ce dernier évoquant le «management sous injonctions paradoxales" pour des institutions transnationales. Cette approche théorique sert aussi d'appui pour analyser la relation des individus avec différentes cultures, dans le cas du management interculturel (Gauthey, 2002). Les points de focalisation de ces différents travaux sont les relations individu-organisation et le modèle organisationnel-modèle interculturel, où les injonctions paradoxales sont aussi observées. Comme le souligne Gauthey (2002), l'interculturel, développé dans les organisations économiques et internationales à travers un message centré sur un style d'organisation ouvert aux "contextes culturels ", ne masquerait-il pas l'intérêt stratégique de l'organisation face à la compétition? Il écrit à ce propos : «Curieux d'observer combien certains discours sur les cultures et l'interculturel véhiculent en fait, le discours du "même"! Un paradoxe 
qui serait à interroger» (2002, p. 4). Le modèle de l'interculturel, tel que décrit, est porteur de contradictions car il cherche "à plus ou moins unifier, plaquer. Cette multiplicité de strates organisationnelles, de logiques identitaires peut être violente et s'apparenter pour les acteurs à des situations de doubles contraintes... Le problème, c'est que déjà sans les dimensions interculturelles, les pratiques des ressources humaines dans les organisations aujourd'hui sont porteuses d'injonctions contradictoires » (ibid., p. 5)

Comment se situe le monde du tourisme-hôtellerie-loisirs dans ce contexte? Le tourisme comme produit et expression des transformations sociétales se présente avec un double visage: un phénomène social et culturel (expériences), mais aussi une activité économique (services et mise en tourisme des territoires). On peut reconnaître certains ingrédients de la double contrainte au sein du tourisme, notamment: la perception binaire (le touriste-le voyageur), un objet en partage (réel-imaginaire), un statut scientifique controversé (discipline-champ d'études) (Kadri, 2008, 2011).

On retrouve aussi certains de ces aspects dans le management de l'organisation touristique, notamment s'agissant de la gestion des ressources humaines soumise à des enjeux et des contraintes : précarité et conditions de l'emploi, mobilité des salariés, culture d'identité non identifiée, interculturel, problème de reconnaissance des acquis, etc. Ces contraintes liées à la gestion de la ressource humaine peuvent aussi devenir des injonctions paradoxales : par exemple, la mobilité est une contrainte mais elle est aussi perçue comme une compétence qu'il s'agit de cultiver ; cela renforce l'idée que si l'on veut progresser dans le monde professionnel, il faudrait accepter la mobilité professionnelle comme un projet individuel et de management, et ainsi organiser sa vie professionnelle en termes de flexibilité, cela pour satisfaire le management qui s'organise à travers les TI et répond à ses enjeux stratégiques, alors que l'individu continuera à faire face à des enjeux professionnels contraignants. Comme le montrait Gauthey (2002), alors que l'entreprise recherche l'efficacité stratégique, elle met aussi en place des méthodes (management participatif, coaching, développement personnel, audit organisationnel, analyse des conflits, etc.). Ce sont ces modes de management qui sont questionnés (adaptation au mode participatif) pour tenter de sortir de la double contrainte. À ce propos, Gauthey (ibid., p. 7), écrit : «De nombreuses entreprises ont attendu de leurs salariés une ouverture, une adaptation aux contraintes mais bien peu de choses qui se fassent de manière cadrée, régulée ». En fait, il s'agirait de développer un mode d'adaptation nécessaire à l'exigence d'adaptation à une pratique managériale (par exemple, le mode participatif, ou celui lié à l'interculturel).

\section{Conclusion}

L'analyse du discours de la GRH en tourisme, à travers des écrits publiés dans la revue Espaces tourisme \& loisirs, est une opération sensible, nécessitant d'aller au-delà d'une simple analyse thématique et de compléter cette dernière par un second processus plus proche de la conceptualisation et de la construction scientifique. En effet, la catégorisation va plus loin que la simple analyse de discours, puisqu'elle permet de déconstruire mais aussi de viser l'émergence de la signification et donc d'opérer des interprétations (Paillé et Muchielli, 2012).

Ce processus de construction sociale des concepts scientifiques, indispensable pour les sciences sociales et notamment les études touristiques, est pourtant critiqué du fait de 
sa relation et de sa proximité avec le « relativisme ». Hacking (2017:9), qui pose déjà la question dans le titre sans équivoque de son ouvrage Entre science et réalité. La construction sociale de quoi?, évoque d'emblée l'importante querelle qui anime la communauté scientifique :

L'idée de construction sociale est, parmi beaucoup d'autres, une idée farouchement combattue dans le cadre des guerres culturelles américaines [...]. L'expression est devenue code. Si vous l'utilisez positivement, vous vous taxez vous-même d'un certain radicalisme. Si vous la dénigrez, vous vous déclarez rationnel, raisonnable et respectable.

$\mathrm{Au}$ fil de ce travail de construction scientifique en études touristiques, notre intérêt portait sur une interrogation: comment appréhender le discours en ressources humaines dans le monde du tourisme? Nous supposions que la situation complexe de cette activité professionnelle et économique, souvent mise en avant dans les écrits, représenterait un facteur important dans la compréhension dudit discours. Aussi l'utilisation de deux types d'analyse de discours, à travers les 63 textes analysés extraits de la revue Espaces tourisme \& loisirs, nous a permis de tirer quelques conclusions :

- L'analyse NVivo, grâce à deux importants regroupements des mots, a montré que la gestion des ressources humaines en tourisme est une fonction qui devra s'adapter continuellement à une situation particulière, marquée à la fois par les contraintes (précarité, saisonnalité, mobilité), par une exigence d'adaptation constante pour répondre aux stratégies de l'organisation (visibilité de la marque, nouvelles valeurs, nouvelles technologies, mobilité interculturelle) ainsi que par la valorisation des qualifications et des métiers, notamment chez les jeunes.

- L'analyse des axes thématiques a permis de venir renforcer ces premières observations, en délimitant une situation double: une partie dominée par des conditions d'emploi difficiles (1/3) et l'autre partie (2/3) faite d'exigences d'adaptation (mobilité internationale, réseaux professionnels, repenser l'organisation du travail, penser la mobilité non comme un frein mais plutôt comme une opportunité). Si la première représente seulement un tiers du noyau, elle comporte néanmoins une contrainte majeure handicapant la situation générale, où prédomine le recours à la pratique professionnelle pour répondre aux exigences d'adaptation. Aussi, nous observons qu'une part importante des pratiques professionnelles managériales est réalisée par des cabinets-conseil (52\%) et des grandes entreprises. Toutefois, ces grandes entreprises peuvent jouer un rôle indéniable dans la diffusion de pratiques managériales novatrices pour l'entreprise. Enfin, signalons que très peu d'études ou d'observations par les milieux universitaires et de recherche sont engagées.

Mais cette analyse thématique reste trop partielle pour parvenir à une déconstruction du discours, d'où la nécessité d'appliquer une autre analyse, celle des catégories conceptualisantes, qui nous permet d'appréhender une réalité sociale complexe et donc de relever une signification et une interprétation du processus lié à cette réalité. Il s'agit conséquemment d'une construction sociale de cette réalité, qui fait émerger non seulement des catégories conceptuelles (cf. tableau 7), mais montre aussi que la réalité touristique est composée d'un noyau double; il s'agit de la double contrainte adaptative, formée de deux éléments exprimant une réalité contraignante: une contrainte situationnelle permanente vécue à la fois par les employés et la hiérarchie managériale et une vision liée à l'adaptation au changement.

50 Le monde de l'activité touristique (tourisme-hôtellerie-loisirs), observé à travers le discours sur la gestion des ressources humaines, serait-il plus soumis à la double contrainte que d'autres secteurs d'activité économique? Si des études sur 
l'organisation ont été réalisées (management interculturel, développement organisationnel, etc.) depuis plus de quatre décennies, nous n'avons pas connaissance de telles observations dans le secteur en tourisme; toutefois des interventions relatives au coaching et à l'audit organisationnel sont indentifiables dans le discours de la GRH pour ce même secteur Ces dernières interventions en tourisme peuvent représenter un matériau important dans l'évaluation de l'importance de la double contrainte, en les complétant d'autres interventions de recherche.

51 Il serait très utile de s'intéresser aux textes sur la gestion des ressources humaines en tourisme parus dans les revues classées, tant francophones qu'anglophones. Dans le contexte francophone, on trouve peu de textes. Par exemple, la revue Gestion des ressources humaines propose peu de titres consacrés au tourisme (deux articles sur deux décennies), alors que la revue Human Resource Management, classée huitième par le Centre national de la recherche scientifique (CNRS), fait référence à plus de 1300 articles portant sur ce secteur, dont près de 1000 en hôtellerie.

52 Les textes de la revue Espaces tourisme \& loisirs sur la gestion des ressources humaines, loin de présenter une vision de l'organisation réduite à la réalité professionnelle (pratiques) de l'activité touristique, constituent un matériau incontournable pour l'identification de deux aspects au plan épistémologique. D'une part, à travers l'analyse du discours et la théorisation du contenu, ce matériau nous convie à percevoir une autre réalité professionnelle, moins visible. Comme l'exprime Wittezaele $(2008: 18)$ à propos de l'intervention de Bateson sur la relation de communication par la théorie de la double contrainte, ce dernier met à jour une autre réalité de la communication et "nous invite à voir l'invisible, en quelque sorte, c'est-à-dire les règles implicites qui régissent les rapports entre les différentes personnes [...]». D'autre part, certains éléments conceptuels de la sociologie du travail ne semblent plus adaptés au contexte de la réorganisation actuelle du travail. Par exemple, le concept de précarité, caractérisant notamment les conditions d'emploi en tourisme, est aujourd'hui réinterrogé, à la faveur d'un concept plus opératoire et moins normatif, comme celui de vulnérabilité (Vultur, 2019). Le monde du tourisme-hôtellerie-loisirs apparait donc comme un milieu spécifique, particulièrement favorable à l'observation de la redéfinition des concepts en sciences sociales.

\section{BIBLIOGRAPHIE}

Gregory BATESON, Vers une écologie de l'esprit, tome 2, Seuil, 1980.

Gregory BATESON, Don D. JACKSON, Jay HALEY et John WEAKLAND, « Toward a theory of schizophrenia », Behavioral Science, 1(4), 1956.

Franck BOURNOIS et C. Brooklyn DERR, « Les directeurs des ressources humaines ont-ils un avenir ? ». Revue française de gestion, $n^{\circ}$ 98, 1994.

Franck BOURNOIS, Jacques ROJOT et Jean-Louis SCARINGELLA, RH, les meilleures pratiques des entreprises du CAC 40, Éditions d'Organisation, 2003. 
Michel CASTEIGTS, « Le pilotage des politiques européennes : un management sous injonctions paradoxales. Ou pourquoi l'Europe est de plus en plus schizophrène. », dans Joachim BECK et Fabrice LARAT (dir.), Transnationale Verwaltungskulturen in Europa - Les cultures administratives transnationales en Europe, Dike Verlag \& Nomos Verlag, 2015 [https://halshs.archives-ouvertes.fr/ halshs-01585711/document].

Cécile CleRgeau, Olivier glasberg et Philippe violier, Management des entreprises du tourisme, Dunod, 2014.

Roy A. соOK, Cathy H.C. HSU et Joseph J. MARQUA, Tourism. The business of hospitality and travel, Pearson, 2014.

Chris COOPER et C. Michael HALL, Le tourisme aujourd'hui : une approche internationale, De Boeck, 2008.

Nicole CôTÉ, Laurent BÉLANGER et Jocelyn JACQUES, La dimension humaine des organisations, Édition G. Morin, 1994.

Michel CROZIER, L'entreprise à l'écoute, InterÉditions, 1989.

Claude DUTERME, « Double contrainte et entreprise : contexte global, paradoxes locaux, souffrances individuelles », dans Jean-Jacques WITTEZAELE (dir.), La double contrainte : l'influence des paradoxes de Bateson en sciences humaines, De Boeck, 2008.

Claire DUPONT, « Du discours stratégique à la construction de la fonction RH par les praticiens », XXII Conférence internationale de management stratégique, 2013.

Franck GAUTHEY, « Management interculturel : représentations et pratiques en questions », Communication et organisation, $\mathrm{n}^{\circ}$ 22, 2002 [http://communicationorganisation.revues.org/2721]

Charles R. GOELDNER et J.R. Brent RITCHIE, Tourism. Principles, practices, philosophies, John Wiley \& Sons, 2009.

Francis GUÉRIN et Frédérique PIGEYRE, « Peut-on encore parler de "la" professionnalisation de la GRH ? ", Actes du $18^{\mathrm{e}}$ congrès de l'AGRH, 2007.

Salvador JUAN et A. ЈАСОВ, Organisation et management en question, coll. « Logiques sociales », L'Harmattan, 1987.

Darren LEE-ROSS et Jospehine PRYCE, Human resources and tourism. Skills, culture and industry, Channel View Publications, 2010.

Erick LEROUX et Pierre-Charles PUPION, Management du tourisme et des loisirs, Vuibert, 2014.

Jean-Pierre LOZATO-GIOTART, Erick LEROUX et Michel BALFET, Management du tourisme. Territoires, offres et stratégies, Pearson, 2012.

Boualem KADRI, « Réflexion sur l'épistémologie du tourisme. La perspective constructiviste », dans Lucie K. MORISSET, Bruno SARRASIN et Guillaume ÉTHIER (dir.), Épistémologie des études touristiques, Presses de l'Université du Québec, 2011.

Boualem KADRI, «L'identité scientifique du tourisme : un mythe ou une réalité en construction », Téoros. Revue de recherche en tourisme, vol. 27, $\mathrm{n}^{\circ} 1,2008$.

Juan MADERA, Mary DAWSON, Priyanko GUCHAIT et Amanda BELARMINO, « Strategic human resources management research in hospitality and tourism : a review of current literature and suggestions for the future ", International journal of contemporary hospitality management, vol. 29, n 1, 2017.

Daniel MERCURE et Mircea VULTUR, Dix concepts pour penser le nouveau monde du travail, Presses de l'Université Laval, 2019. 
Alain MESPLIER et Pierre BLOC-DURAFFOUR, Le tourisme dans le monde, Bréal, 2014.

Michel MONEREAU, Management des organisations touristiques, Bréal, 2008.

Pierre PAILLÉ et Alex mucCHIELLI, L'analyse qualitative en sciences humaines et sociales, Armand Colin, 2008.

Jean-Marie PERETTI, Ressources Humaines, Vuibert, 2013.

Michel Lussault et Mathis STOCK, « Tourisme et urbanité ", dans Philippe DUhamel et Rémi KNAFou (dir.), Les mondes urbains du tourisme, Belin, 2007.

Stephen ROBBINS et Timothy JUDGE, Les comportements organisationnels, $14^{\mathrm{e}}$ éditions, Pearson Education, 2011.

Mathis STOCK, Olivier DEHOORNE, Philippe DUHAMEL, Jean-Christophe GUAY, Rémy KNAFOU, Olivier LAZZAROTI, Isabelle SACAREAU et Philippe VIOLIER, Le tourisme : acteurs, lieux et enjeux, Belin, 2003.

Yves TINARD, Le tourisme économie et management, McGraw-Hill, 1992.

Mircea VULTUR, « Le concept de précarité : quelle adéquation aux transformations actuelles du monde du travail », dans Daniel MERCURE et Mircea VULTUR (dir.), Dix concepts pour penser le nouveau monde du travail, Presses de l'Université Laval, 2019.

Paul WATZLAWICK, Janet HELMick et Don de Avila JACKSON, Une logique de la communication, Points Essais, 1972.

Jean-Jacques WITTEZAELE, « La double contrainte ; un concept fondateur », dans Jean-Jacques WITTEZAELE (dir.), La double contrainte : l'influence des paradoxes de Bateson en sciences humaines, De Boeck, 2008.

\section{RÉSUMÉS}

L'entreprise, acteur majeur dans la société aujourd'hui, fait de plus en plus l'objet d'une attention particulière des chercheurs et managers d'entreprises, à travers notamment l'analyse de la fonction des ressources humaines. L'industrie du tourisme et des loisirs, qui montre une grande particularité de sa ressource humaine (importante mobilité, partie intégrante de l'expérience), fait observer une perspective pratique et professionnelle plus développée que l'appréhension scientifique. Si le discours scientifique sur cette complexité touristique est bien constaté, en est-il de même du discours concernant la gestion des ressources humaines? Dans cet article, nous analysons le discours en gestion des ressources humaines dans la recherche en tourisme, notamment dans les ouvrages et les revues. La perspective proposée est une interrogation de type épistémologique privilégiant une approche de type constructiviste pour l'analyse du discours des chercheurs. Deux analyses sont développées : d'une part, l'analyse thématique pour faire faire émerger les thèmes pertinents d'un corpus; d'autre part, l'analyse des «catégories conceptualisantes", afin d'apporter les premiers éléments de théorisation d'un phénomène. Cette analyse de la gestion des ressources humaines en tourisme est réalisée à travers les écrits parus dans la revue francophone Espaces tourisme \& loisirs, qui permet d'apporter un regard diachronique sur l'évolution du discours en gestion des ressources humaines, et ce, depuis les années 1990.

The company, a major player in society today, is increasingly the subject of particular attention from researchers and business managers, notably through the analysis of the human resources function. The tourism and leisure industry, which shows a great particularity of its human 
resources (significant mobility, an integral part of the experience), points out a practical and professional perspective more developed than scientific apprehension. As such, this paper attempts to understand how the discourse in human resources management in tourism research looks like, especially in books and journals. In order to answer this question, the proposed perspective is an epistemological type of questioning favoring a constructivist approach to the analysis of the researchers' discourse. Two analyzes are developed : first, a thematic analysis to bring out the relevant themes from a corpus; second, the analysis of "conceptualizing categories", in order to provide the first elements of theorizing a phenomenon. This analysis of human resource management in tourism is carried out through the writings published in the French-language journal Espace tourisme \& loisirs, which provides a diachronic view of the evolution of the discourse on human resource management since the 1990s.

INDEX

Mots-clés : tourisme, ressources humaines, emploi, théorie du management

Keywords : human resources, employment, tourism, management theory

\section{AUTEURS}

\section{BOUALEM KADRI}

$\mathrm{PhD}$

kadri.boualem@uqam.ca

\section{MOHAMED REDA KHOMSI}

$\mathrm{PhD}$

khomsi.mohamed_reda@uqam.ca

\section{CYRIL MARTIN}

martin.cyril@uqam.ca 